\title{
Desempeño productivo y conductas etológicas de gallinas ponedoras en tres tipos de manejo en condiciones de trópico cálido
}

\author{
Productive performance and behavior of laying hens in three housing systems \\ under tropical conditions
}

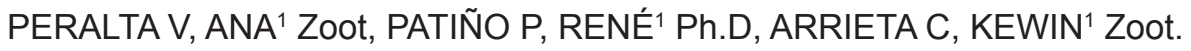

${ }^{1}$ Universidad de Sucre, Facultad de Ciencias Agropecuarias, Sincelejo - Colombia.

\section{Keywords:}

Animal well-being; ethology;

housing systems; laying hens.

\begin{abstract}
The objective of this study was to evaluate the effect of management systems (T1:cage, T2:litter floor and T3:free range) on productive performance and behavior of laying hens of a commercial line in a hot tropical environment conditions, 220 laying hens of Lohmann Brown line with 56 weeks of age were randomly assigned to three treatments. Physiological, anatomical, behavioral and productive variables were studied. Caged birds had a higher incidence of ridges, chin, legs and plumage with undesirable characteristics, indicator for the housing problems, like increased mortality $(\mathrm{P}=0.002)$. The highest eggs production $(79.9 \%)$ was observed in litter floor management, with no effect $(P>0.05)$ of management systems on egg weight and morbidity. Birds kept in cage spent more time $(P<0.05)$ feed intake. Hens managed in grazing and spent more time on floor $(\mathrm{P}<0.05)$ stretching activities, and "bath" with sand or floor material. It is concluded that the handling of hens in litter floor promoted a better productive performance and facilitated the expression of own behavior of these birds, while handling cages negatively affected bird performance, appearance and welfare.
\end{abstract}

\section{Resumen}

El objetivo del estudio fue evaluar el efecto del tipo de manejo (T1:jaula; T2:piso y T3:pastoreo) sobre variables de tipo etológico, fisiológico y de desempeño productivo en gallinas de postura en condiciones de trópico, para lo cual, 220 aves de la línea Lohmann Brown con 56 semanas de edad fueron asignadas aleatoriamente a los tres tratamientos (tipo de manejo), para evaluar variables fisiológicas, anatómicas, productivas y etológicas. Las aves enjauladas presentaron mayor frecuencia (\%) de crestas, barbilla, plumaje y patas con características no deseables, indicadoras de problemas durante el alojamiento, al igual que mayor mortalidad $(P=0,002)$. Las gallinas manejadas en piso presentaron el mayor porcentaje de postura $(79,9 \%)$; el tipo de manejo no afectó $(P>0,05)$ ni el peso del huevo ni la morbilidad. Las aves en jaula dedicaron más tiempo $(P<0.05)$ al consumo de alimento. Las gallinas en pastoreo y las manejadas en piso dedicaron más tiempo $(P<0,05)$ a actividades de estiramiento, y "baños" con arena o material del piso. Se concluye que el manejo de gallinas en piso promovió un mejor desempeño productivo y facilitó la expresión de conductas propias de estas aves, mientras que el manejo en jaulas afectó negativamente el desempeño de las aves, la apariencia y el bienestar de las mismas. 


\section{Introducción}

En varios países del mundo, principalmente en Europa, se está trabajando en la redefinición de los estándares de producción basados en los criterios del bienestar animal, para atender nuevas normatividades (ESTRADA et al., 2015). Existen básicamente tres sistemas de manejo de gallinas ponedoras: en jaulas, en piso y libres, o en pastoreo (KEELING, 2003), cada uno con características propias, las cuales, además de impactar el desempeño, inciden sobre el comportamiento o conductas de las aves (YILMAZ et al., 2016). En varios países existen plazos definidos para dejar de usar el sistema de manejo en jaulas, ya que se ha comprobado que las aves manejadas bajo estos sistemas no pueden expresar varias de las conductas propias de la especie, lo que se considera atenta contra el bienestar de las mismas (SOSÓWKA-CZAJKA et al., 2010). Por otra parte, los sistemas de manejo "en libertad" favorecen el bienestar de las aves, pero existen resultados diversos en relación a la productividad bajo esas condiciones, debido a las variaciones presentadas en los mismos (LAY et al., 2011), las cuales prevalecen en condiciones trópico bajo. El objetivo del presente estudio fue evaluar el efecto de tres tipos de manejo (jaula, piso y pastoreo) sobre el desempeño productivo y conductas etológicas de gallinas de postura de una línea comercial en condiciones de trópico cálido.

\section{Materiales y métodos}

Ubicación. Este trabajo fue desarrollado en la granja experimental "los Pericos", de la Universidad de Sucre, en el municipio de Sampués - departamento de Sucre, a $9^{\circ} 15^{\prime}$ Norte y $71^{\circ} 22^{\prime} 54^{\prime \prime}$ Oeste; a una altura de 202 m.s.n.m., en zona perteneciente al bosque seco tropical (HOLDRIDGE, 1967). El local se encuentra en el piso térmico cálido, con una temperatura que oscila entre $21-32^{\circ} \mathrm{C}$, con humedad relativa del $75 \%$ en promedio.

Tratamientos e infraestructura. Se evaluaron tres tipos de manejo, los cuales conformaron los tratamientos del estudio: T1-Jaula; T2-Piso y T3Pastoreo. Los tratamiento T1 y T2 se efectuaron en un mismo galpón, que contaba con techo de asbesto y un cierre perimetral en malla metálica. En el T1 las aves se alojaron en una batería de 54 jaulas de plástico individuales, distribuidas en tres niveles $(35 \mathrm{~cm}$ de alto $X 20 \mathrm{~cm}$ de ancho $X$ $38 \mathrm{~cm}$ de largo, área $=760 \mathrm{~cm} 2)$ (18 jaulas/nivel). El área de cada jaula fue de $760 \mathrm{~cm}^{2}$. La densidad de aves usada en los tratamientos T2 y T3 fue de 6 aves $/ \mathrm{m}^{2}$ y de 0.17 aves $/ \mathrm{m}^{2}$.

Las aves del T2 se confinaron en un área de 12,96 m2, dividida en tres lotes separados por malla metálica $\left(4,32 \mathrm{~m}^{2}\right.$ cada área), provista de un piso de cemento y cama de viruta de madera. Cada área se dotó de un módulo de cinco nidos elaborados en madera y plástico $(1,50 \mathrm{~m}$ de frente $\times 0,38 \mathrm{~m}$ de fondo $\times 0,30 \mathrm{~m}$ de altura), un comedero semiautomático tipo tolva y dos bebederos automáticos tipo campana. Las aves del T3 se alojaron en tres corrales $(28,9 \mathrm{~m}$ de largo $\times 5,2 \mathrm{~m}$ de ancho, con área total de $\left.450,84 \mathrm{~m}^{2}\right)$, provistos de cercas vivas de matarratón (Gliricidia sepium), totumo (Crescentia cujete) y guayaba dulce (Psidium guajava) y cierre perimetral en malla plástica. Cada área contó con una caseta de madera $\left(2 \mathrm{~m}^{2}\right)$, con techo de tela, en el cual se localizó un comedero tipo tolva de material plástico y un bebedero de plástico. Así mismo se localizó un módulo de cinco nidos con las mismas características del utilizado en el T2, una percha elaborada de madera de $1,8 \mathrm{~m}$ de largo $\times 0,8 \mathrm{~m}$ de alto.

Unidades experimentales. Se emplearon 220 gallinas de la línea Lohmann Brown Classic, (56 semanas de edad y $1.884 \mathrm{~g}$ de peso). Las aves fueron seleccionadas al azar para cada uno de los tres tratamientos y se inició el experimento después de siete días de adaptación de las aves al manejo. En el T1 se asignaron 53 aves, distribuidas en 18 jaulas por nivel. Para el T2 se asignaron 26, 25, y 26 aves en cada lote respectivamente. En el T3 se utilizaron tres lotes de 30 aves cada uno. Se efectúo un muestreo estratificado de 12 aves por tratamiento, las cuales se marcaron individualmente en la pata izquierda con cintas de color diferente por lote. En estas 36 aves se evaluaron las variables fisiológicas, conductuales y fenotípicas; así como el peso vivo. Las evaluaciones se realizaron entre las semanas 56 y 72 de vida de las aves. 
Manejo alimenticio. Las aves de todos los tratamientos fueron alimentadas con alimento balanceado comercial específico para la etapa, el cual fue suministrado desde el inicio de la fase de postura. Las gallinas de los tratamientos T1 y T2 recibían el alimento como dieta sólida única a las 08:00 horas, a razón de $105 \mathrm{~g} \mathrm{día}^{-1}$, mientras que a las aves en pastoreo recibían $63 \mathrm{~g} \mathrm{día}^{-1}$, valor correspondiente al $60 \%$ de la oferta en los tratamientos T1 y T2, debido a que la dieta se complementó con alimento presente en la zona de pastoreo, en la cual predominó la gramínea Botriochloa pertusa. La cantidad ofrecida era ajustada en caso de presentarse mortalidad.

Durante la fase experimental se tomaron dos muestras representativas del pasto ofrecido a las aves manejadas en pastoreo y del balanceado comercial para corroborar su composición nutricional, a partir de la determinación de los porcentajes de materia seca, cenizas, proteína bruta y extracto etéreo, según AOAC (1990). Las determinaciones se llevaron a cabo en el laboratorio de Nutrición Animal de la Universidad de Sucre. Los valores de la composición química del alimento balanceado comercial correspondieron a los ofrecidos por el fabricante, mientras la pastura en la que se manejaron las aves en pastoreo presentó $26 \%$ de materia seca; $10,4 \%$ de proteína bruta; $11,8 \%$ de cenizas y $3,2 \%$ de extracto etéreo.

Variables en estudio. Las variables estudiadas fueron:

Temperatura corporal y frecuencia respiratoria: La medición de la temperatura corporal y la frecuencia respiratoria se realizó una vez por semana, a las a las 08:00 y a las 16:00 h. Para la medición de la temperatura se utilizó un termómetro rectal durante dos minutos y la frecuencia respiratoria mediante el conteo de los movimientos de jadeo durante 15 minutos (BARBOSA, 2004).

Coloración y tamaño de crestas y barbillas, y caracterización de patas: Las observaciones realizadas en crestas, barbillas y patas se realizaron semanalmente durante todo el periodo experimental. Para evaluar la coloración de crestas y barbillas, además del tamaño de las mismas, se estableció una escala con tres tipos de colores (pálida, rosada y roja) y tamaños (pequeña, mediana y grande), teniendo como referencia la coloración y tamaño observado al inicio de la fase de toma de datos. En el caso de las patas se observó coloración, largo de uñas, parte plantar, forma de dedos, grosor y descamaciones de las mismas.

Porcentajes de mortalidad y morbilidad: Se registró durante todo el estudio la mortalidad, la presencia de aves enfermas, el total de huevos colectados, teniendo en cuenta el número de aves con el que se inició, para posteriormente calcular el valor porcentual en cada caso.

Variación del peso corporal: Los pesajes de las aves se realizaron semanalmente, a las 08:00 h., antes de la oferta de alimento. En total se realizaron 17 pesajes, usando una báscula colgante doble reloj con platón (modelo TECNIPESA BC6). EI primer pesaje se consideró como referente para el cálculo de la pérdida o ganancia de peso durante el periodo experimental.

Peso del huevo y porcentaje de postura: Se contabilizaron y pesaron la totalidad de huevos colectados. A partir del número de huevos, y considerando el número de aves presentes se calculó el porcentaje de postura.

Variables de tipo etológico: Se hizo un muestreo focal de acuerdo con el protocolo propuesto por WELFARE QUALITY (2009). Las mediciones las efectuaron ocho evaluadores entrenados dos veces por semana durante 28 días de estudio, en los períodos de luminosidad, de manera simultánea en los tres tratamientos. Las observaciones se realizaron cada cinco minutos para establecer el tiempo dedicado a realizar actividades como: comer, beber, investigar plumas, tomar "baños" de arena, estirar y agitar plumas, escarbar, buscar nido, y la posición (sentada o echada) (BARBOSA, 2004), de igual manera se evaluaron conductas agresivas. A la conducta registrada en el momento de la observación se le registró un tiempo igual a cinco minutos, para totalizar en minutos/día.

Diseño experimental y análisis de los datos. Los datos de las variables peso corporal, conductas (variables de tipo etológico), frecuencia respiratoria y temperatura corporal se analizaron según un diseño completamente al azar con medidas repetidas en el tiempo. El análisis de varianza se llevó a cabo utilizando el Software 
IBM SPSS Statistics (V.P). Se verificó el supuesto de homocedasticidad y normalidad de los datos, usando las pruebas de Levenne y Shapiro Wilk, respectivamente. Se aplicó la prueba de esfericidad de Mauchy que contrastó la hipótesis nula de que la matriz de covarianza del error de las variables dependientes transformadas era proporcional a una matriz identidad. Los efectos entre-sujetos correspondieron a los tratamientos (tipos de manejo), y los efectos intra-sujetos a los días en que se realizaron las observaciones. Las medias de cada variable fueron comparadas usando la prueba de Tukey, a nivel de significancia de $0.05 \%$. En el caso de las variables relacionadas con el desempeño productivo (peso del huevo y porcentaje de postura), se consideró cada día como réplica, en cada uno de los tratamientos. En el caso de la caracterización de crestas, barbilla y patas, solamente se procedió a cuantificar la mayor cantidad de casos, para caracterizar cada tipo de manejo, al finalizar el periodo de observación, de manera que se identificara cada tipo de manejo con una característica principal en relación a color y/o forma.

\section{Resultados}

En relación a la frecuencia respiratoria y a la temperatura corporal se encontró que las aves manejadas en pastoreo presentaron mayor $(P<0,001)$ frecuencia respiratoria, tanto en las horas de la mañana como en la tarde (Tabla 1), mientras que la temperatura corporal no fue influenciada por los tratamientos $(P>0,05)$.
En la Figura. 1, se observa la coloración predominante en crestas y barbillas de las aves. Se nota que la coloración en las aves enjauladas se presentó entre pálida a muy pálida y seca, en piso predominaron las rosadas y en pastoreo las coloraciones rojas, hasta rojo intenso y se notaron suaves al tacto.

Las patas de las aves enjauladas se observaron limpias, muy delgadas, de coloración amarillo pálido a blanco, uñas muy largas. Los dedos se apreciaron deformes, lo que impedía el normal desplazamiento de las mismas. Por otra parte, las aves manejadas en piso presentaron lesiones plantares. En pastoreo se observaron uñas cortas. El plumaje de las aves enjauladas se notó deteriorado en comparación a las gallinas de los otros sistemas de manejo.

Tabla 1. Efecto de tres tipos de manejo sobre la frecuencia respiratoria (respiraciones/min.) y la temperatura corporal $\left({ }^{\circ} \mathrm{C}\right)$ durante la mañana $(\mathrm{am})$ y la tarde (pm) en gallinas de postura de la línea Lohmann Brown en condiciones de trópico cálido.

\begin{tabular}{cccccc}
\hline \multirow{2}{*}{ Variables } & \multicolumn{3}{c}{ Tratamiento $^{1}$} & & \\
\cline { 2 - 5 } & Jaula & Piso & Pastoreo & & \\
\hline Frecuencia respiratoria (am) & $29,6 \mathbf{a}$ & $29,2 \mathbf{a}$ & $31,3 \mathbf{b}$ & 0,001 & 0,30 \\
& & & & & EE$^{2}$ \\
Frecuencia respiratoria (pm) & $27,5 \mathbf{a}$ & $28,5 \mathbf{a}$ & $30,3 \mathbf{b}$ & 0,001 & 0,41 \\
Temperatura (am) & $40,35 \mathbf{a}$ & $40,35 \mathbf{a}$ & $40,29 \mathbf{a}$ & 0,876 & 0,10 \\
Temperatura $(\mathrm{pm})$ & $40,4 \mathbf{a}$ & $40,3 \mathbf{a}$ & $40,27 \mathbf{a}$ & 0,408 & 0,05 \\
\hline
\end{tabular}

${ }^{1}$ Medias seguidas de letras diferentes en las filas difieren estadísticamente según la prueba de Tukey al $5 \%$ de probabilidad. ${ }^{2}$ Error estándar de la media.
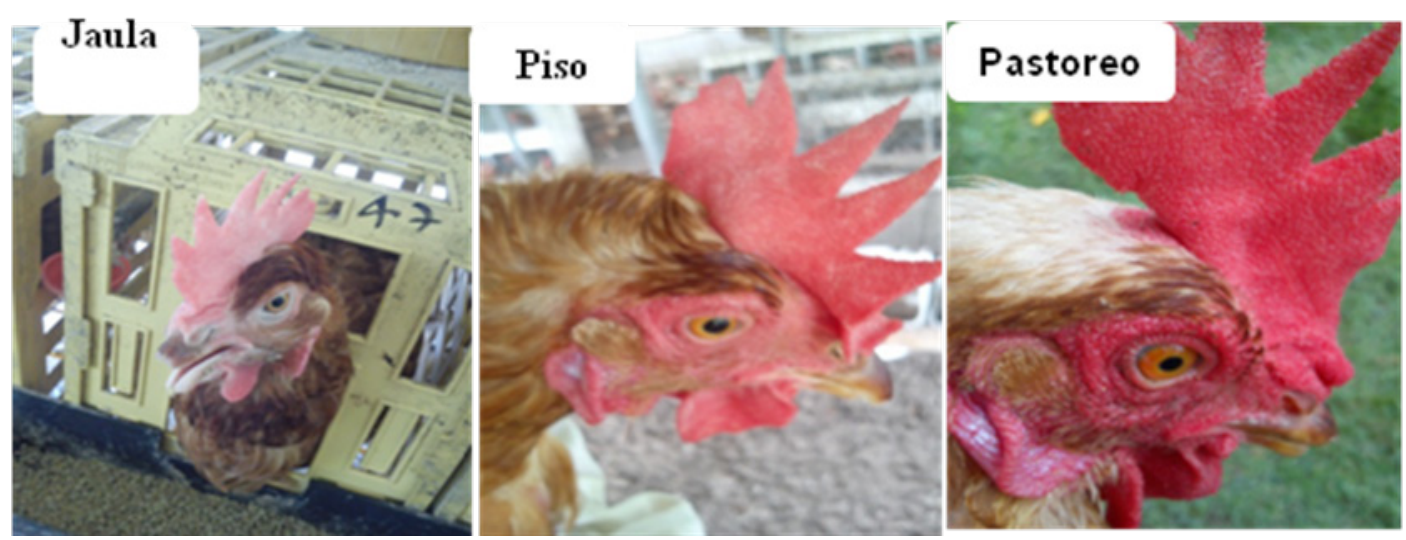

Figura 1. Efecto de tres tipos de manejo sobre la coloración de cresta y barbillas en gallinas de postura de la línea Lohmann Brown en condiciones de trópico cálido. 
No se presentaron diferencia entre tratamientos en relación al peso del huevo $(P=0,140)$, el cual presentó un valor medio de 63,6 g (Tabla 2).

Tabla 2. Efecto de tres tipos de manejo sobre variables indicadores de desempeño productivo en gallinas de postura de la línea Lohmann Brown en condiciones de trópico cálido.

\begin{tabular}{cccccc}
\hline \multirow{2}{*}{ Variable } & \multicolumn{3}{c}{ Tratamiento $^{1}$} & Valor de P & EE $^{2}$ \\
\cline { 2 - 5 } & Jaula & Piso & Pastoreo & & \\
\hline Peso huevo entero, g & $61,1 \mathbf{a}$ & $66,7 \mathbf{a}$ & $63,1 \mathbf{a}$ & 0,140 & 1,12 \\
Postura, \% & $38,0 \mathbf{a}$ & $79,9 \mathbf{c}$ & $54,1 \mathbf{b}$ & $<0,001$ & 0,96 \\
Morbilidad, \% & $0,01 \mathbf{a}$ & $\mathbf{0 a}$ & $0,06 \mathbf{a}$ & 0,116 & 0,02 \\
Mortalidad, \% & $0,19 \mathbf{b}$ & $0 \mathbf{a}$ & $0,05 \mathbf{a}$ & 0,002 & 0,04 \\
$\begin{array}{c}\text { Variación del peso } \\
\text { corporal, g }\end{array}$ & $-127,8 \mathbf{a}$ & $165,0 \mathbf{b}$ & $35,5 \mathbf{a b}$ & 0,033 & 46.7 \\
\hline
\end{tabular}

${ }^{1}$ Medias seguidas de letras diferentes en las filas difieren estadísticamente según la prueba de Tukey al $5 \%$ de probabilidad. ${ }^{2}$ Error estándar de la media.

La mayor mortalidad $(P=0,002)$ se observó en el T1 y no se observaron diferencias $(P>0,05)$ entre T2 y T3. La morbilidad no varió entre tratamientos $(P=0,116)$. El mayor porcentaje de postura $(P<0,001)$ se presentó en las aves del T2, superando a las aves en pastoreo en $25.9 \%$ y a las de jaula en $41.9 \%$.

Al finalizar la fase experimental, las aves de los tratamientos T2 y T3 presentaron un incremento en peso de 165 y $35,5 \mathrm{~g}$ respectivamente, mientras que las gallinas manejadas en jaula (T1) perdieron 127,8 $\mathrm{g}$ durante el periodo.

Las aves enjauladas dedicaron más tiempo $(P=0,022)$ al consumo de alimento balanceado (Tabla 3). El tiempo dedicado al consumo de ración no varió $(P<0,07)$ entre las aves en piso y en pastoreo. No se encontraron diferencias significativas $(P>0,05)$ entre tratamientos en los tiempos dedicados a beber agua, acicalarse, estiramiento de alas, agitación de plumas, escarbar tierra (solo piso y pastoreo), a permanecer echadas o en nido, a las confrontaciones, a permanecer de pie, a picar el suelo o la jaula, a caminar (solo piso y pastoreo) y a estirar las patas.

Las gallinas del T3 dedicaron más tiempo $(P<0,05)$ a estirar las alas, mientras las del T2 más tiempo a los "baños" con la cama. No se apreciaron diferencias entre los tratamientos T2 y T3 $(P>0,05)$ para las conductas agresivas y el tiempo dedicado a caminar (Tabla 3).
Tabla 3. Efecto de tres tipos de manejo sobre variables (valores en minutos por día) de naturaleza etológica indicadoras de bienestar en gallinas de postura de la línea Lohmann Brown en condiciones de trópico cálido.

\begin{tabular}{|c|c|c|c|c|c|}
\hline \multirow{2}{*}{ Variable } & \multicolumn{3}{|c|}{ Tratamiento ${ }^{1}$} & \multirow{2}{*}{ - Valor de $\mathrm{P}$} & \multirow{2}{*}{$\mathrm{EE}^{2}$} \\
\hline & Jaula & Piso & Pastoreo & & \\
\hline $\begin{array}{l}\text { Consumo de alimento } \\
\text { completo }\end{array}$ & $46,0 \mathbf{a}$ & $21,6 \mathbf{b}$ & $18,4 \mathbf{b}$ & 0,022 & 4,38 \\
\hline Consumo de agua & $19,5 \mathbf{a}$ & $10,6 \mathbf{a}$ & $11,5 \mathbf{a}$ & 0,097 & 1,81 \\
\hline Acicalamiento & $9,0 \mathbf{a}$ & $7,6 \mathbf{a}$ & $12,7 \mathbf{a}$ & 0,271 & 1,33 \\
\hline Estiramiento de alas & $0,25 \mathbf{a}$ & $0,73 \mathbf{a}$ & $0,73 \mathbf{b}$ & 0,019 & 0,42 \\
\hline Agitación de plumas & $0,03 \mathbf{a}$ & $0,91 \mathbf{a}$ & $0,39 \mathbf{a}$ & 0,384 & 0,27 \\
\hline Escarbar cama o tierra & - & $10,9 a$ & $9,2 \mathbf{a}$ & 0,789 & 2,91 \\
\hline Echada & $26,1 \mathrm{a}$ & $21,1 a$ & $3,0 \mathbf{a}$ & 0,149 & 4,78 \\
\hline Permanencia en nido & $1,8 \mathbf{a}$ & $3,0 \mathbf{a}$ & $2,4 a$ & 0,808 & 0,72 \\
\hline Confrontaciones & - & $0,07 \mathbf{a}$ & $0,01 \mathbf{a}$ & 0,197 & 0,02 \\
\hline Parada & $67,2 a$ & $66,5 a$ & $47,9 a$ & 0,584 & 7,99 \\
\hline Jadeando & $23,2 a$ & $44,7 \mathbf{a}$ & $31,8 \mathbf{a}$ & 0,475 & 7,30 \\
\hline Picando suelo o jaula & $0,37 \mathbf{a}$ & $11,14 a$ & $2,66 \mathbf{a}$ & 0,073 & 2,14 \\
\hline Caminar & - & $50,4 a$ & $51,9 a$ & 0,889 & 4,76 \\
\hline Estiramiento de patas & - & $0,25 a$ & $0,63 \mathbf{a}$ & 0,533 & 0,28 \\
\hline "Baños" con cama o suelo & - & $5,71 \mathrm{a}$ & $0,04 \mathbf{b}$ & 0,033 & 1,34 \\
\hline
\end{tabular}

${ }^{1}$ Medias seguidas de letras diferentes en las filas difieren estadísticamente según la prueba de Tukey al $5 \%$ de probabilidad. ${ }^{2}$ Error estándar de la media. ${ }^{3}$ Conductas no posibilitadas por la condición de manejo se indican con guion (-).

\section{Discusión}

La frecuencia respiratoria y la temperatura corporal presentaron valores dentro de los rangos normales para en todos los tratamientos, a pesar de la mayor frecuencia observada en el T3 (Tabla 2). Según GARCÍA et al. (2013) valores entre 2540 respiraciones/minuto y entre $40,6-41,7^{\circ} \mathrm{C}$, para la frecuencia respiratoria y la temperatura corporal, respectivamente, son considerados normales. La mayor actividad física de las aves en pastoreo, al igual que el tipo de instalación, podría incrementar el jadeo como mecanismo de termorregulación, como indicado por GARCIA et al. (2009) para aves en ese tipo de manejo.

La coloración de crestas y barbillas se vio influenciada por el tipo de manejo. Se ha descrito que el estrés causado por la permanencia de las aves alojadas en jaulas influye sobre la eficiencia de uso del alimento, lo que puede afectar el cumplimiento de sus requerimientos nutricionales para producir adecuadamente (BASTMAN et al., 2011). En este estudio la presencia de crestas y barbillas pálidas en las aves alojadas en las jaulas pudo estar relacionado con el efecto negativo de 
este sistema, el cual no sólo afectó la producción de huevo, sino que causó mayor mortalidad, cuando se compararon estos criterios con los otros dos sistemas evaluados, aspecto que ha sido descrito por otros investigadores (HINCAPIÉ y RODAS, 2001; BALLINA, 2008) quienes recalcan que en las gallinas enfermas y en las que dejan de poner, la cresta y las barbillas se decoloran, disminuyen de tamaño, y se notan secas y ásperas al tacto.

En cuanto a las características de las patas y uñas también se observaron características diferenciales entre los tipos de manejo evaluados. En cada caso se presentaron características propias correspondientes a las condiciones del piso o estructuras de apoyo para las aves. La coloración más pálida y el menor grosor de patas y mayor proporción de dedos deformes se observó en las aves enjauladas, las cuales además presentaban uñas más largas que afectaban la normal locomoción. Por otra parte, las aves manejadas en piso presentaron lesiones plantares, suciedad acumulada en la base de las uñas, originadas por la humedad que se puede presentar en la cama a la hora de realizar la bebida por parte de las aves, ocasionando la rotura de las uñas y sangrados plantares, coloración rosada y uñas no muy largas. SÁNCHEZ et al. (2011) encontraron diferencias al comparar patas de gallinas enjauladas y manejadas en piso, siendo las primeras de mejor estado que las de piso y observando mutilación de dedos, lesiones plantares, desgaste de uñas y patas. CASTAÑEDA y GÓMEZ (2011), quienes compraron también los tres tipos de manejo, observaron que todas las aves manejadas en jaula presentaron alteraciones en la longitud de uñas, lo que incrementó el número de huevos rotos, mientras que no hallaron diferencias en las gallinas manejadas en piso y pastoreo. En este sentido, BALLINA (2008) afirma que en las gallinas sanas y productivas se observan patas amarillentas, mientras que las gallinas enfermas y en las que dejan de poner, las patas aparecen de color amarillo pálido o blanco, como se observaron en este trabajo en el caso del manejo en jaula.

El peso del huevo, que no varió $(P=0,140)$ entre tratamientos, presentó un valor medio de $63,6 \mathrm{~g}$, equivalente a huevo tipo AA (FENAVI, 2015). En todos los casos los huevos se clasificaron mínimo en el tipo $A$, ya que todos los pesos superaron los $53 \mathrm{~g}$, sin embargo, los huevos tipo AAA predominaron en las aves manejadas en piso. Según la guía de manejo de la casa comercial de línea Lohmann Brown Classic (LOHMANN TIERZUCHT GMBH, 2015), en gallinas después de las 54 semanas se deberían esperar pesos del huevo superiores a los $66.1 \mathrm{~g}$. Las condiciones específicas de la localidad y el tipo de manejo podrían explicar la diferencia en relación a la meta, aunque la diferencia es inferior al $3 \%$.

Durante este lapso no se observaron diferencias $(P>0,05)$ entre tratamientos con respecto a la morbilidad, pero sí $(P<0,05)$ en cuanto a mortalidad y porcentaje de postura. El mayor porcentaje de postura se obtuvo de las aves en piso con $79.91 \%$, superando a los demás tipos de manejo. Se debe destacar el bajo nivel de producción de las aves enjauladas, en coherencia con la pérdida de peso y la condición exterior presentada por las aves enjauladas, a pesar de que la oferta y el consumo de alimento fue igual al de las aves manejadas en piso. CASTAÑEDA y GÓMEZ (2011) observaron mayor porcentaje de postura en gallinas en pastoreo (85,7\%) en comparación a los grupos que permanecieron en piso y jaula. GARCÍA et al. (2013) afirman que para obtener un beneficio aceptable de las gallinas bajo producción ecológica, la puesta debe encontrarse por encima del $60 \%$, valor que en el presente estudio solamente fue superado por las aves en piso. Los valores obtenidos para las aves en pastoreo y en jaula fueron inferiores a los indicados por la guía de manejo (LOHMANN TIERZUCHT $\mathrm{GMBH}, 2015)$, que indican una media esperada de $75 \%$, en las edades evaluadas. Varios factores pueden influenciar negativamente el porcentaje de postura. El estrés causado por el manejo en jaula, y el consumo de alimento de las aves en pastoreo podrían ser aspectos a considerar en este sentido, como lo indican BASTMAN et al. (2011).

El estrés, por la limitación de espacio, podría ser una de las causas, ya que el módulo usado tenía espacios de alojamiento individual y no grupal. Esta limitación no permitía la realización normal de conductas consideradas vitales para este tipo de aves. Estos resultados difieren de los encontrados por CASTAÑEDA y GÓMEZ (2011), quienes encontraron mortalidades de $1.87 \mathrm{y}$ $1.17 \%$ en condiciones de manejo en piso y jaula, 
respectivamente, mientras que en pastoreo la sobrevivencia fue de $100 \%$. En ese estudio, las evaluaciones se realizaron en aves más jóvenes (17-35 semanas), sin embargo los valores de mortalidad fueron superiores.

El peso corporal de las aves al inicio del experimento, fue de $1884 \mathrm{~g}$. Luego de la evaluación, es decir al finalizar la fase experimental, las aves en piso y pastoreo presentaron un incremento $(P=0,033)$ en peso de 165 y $35,5 \mathrm{~g}$ respectivamente, mientras que las gallinas manejadas en jaula perdieron $127,8 \mathrm{~g}$ durante la fase experimental. En este sentido, LASCARRO (2014) indica que aves de este tipo, deben iniciar el ciclo de postura con 1500 - $1600 \mathrm{~g}$ y terminar con 80 semanas en $2150 \mathrm{~g}$. CASTAÑEDA y GÓMEZ (2011) encontraron pesos superiores al finalizar (2100 g), entre la semana 17 y la 35. Según la guía de manejo de la casa comercial (LOHMANN TIERZUCHT GMBH, 2015), los pesos a estas edades deberían superar los $2000 \mathrm{~g}$, lo que indica que las aves manejadas en piso fueron las únicas en alcanzar el peso esperado e indicado por la casa comercial de la línea. Una posible causa del bajo incremento en peso de las gallinas manejadas en pastoreo podría ser la oferta reducida de alimento balanceado, el cual se racionó al $60 \%$ del ofrecido a las aves en piso, considerando posibles aportes de otras fuentes presentes en el sistema en pastoreo. Esta restricción, dada por el modelo de manejo podría no ser la más indicada. El bajo porcentaje de postura podría estar asociado al mismo hecho. Según LI et al. (2011), la reducción en el consumo de alimento causa reducción en la producción de huevo, y en el tamaño, sin embargo, en el presente estudio no se encontraron diferencias en el peso del huevo en los diferentes tratamientos.

El mayor tiempo de permanencia $(P=0,022)$ en comedero por parte de las aves enjauladas se puede explicar por la posición de las aves en la jaula y por la posición del comedero. La inhabilidad para realizar otras actividades impedía la realización de otras conductas, por lo que la actividad en comedero era la principal conducta realizada. Las aves en pastoreo sí recibían menos cantidad de alimento balanceado, siguiendo un modelo propuesto de manejo para ese tipo de manejo, lo que coincide con lo indicado por CASTELLÓN et al. (2013), quienes afirman que las gallinas dedican el $30 \%$ de su tiempo a beber y alimentarse ya sea búsqueda del alimento.
La imposibilidad de las aves enjauladas para realizar ciertas conductas básicas, como la de caminar, por ejemplo, es indicativo de falta de bienestar, como lo indican KEELING (2003) y CASTELLÓN et al. (2013), quienes afirman que el diseño de las instalaciones y la disponibilidad de las mismas son de gran importancia a la hora de proteger la higiene, el bienestar y el rendimiento productivo de las aves. Según BARBOSA (2004), cuando las aves se echan sobre el suelo pueden realizar termorregulación y lo normal es que lo realicen antes de la postura de un huevo.

La presencia de conductas agresivas entre aves no fue afectada $(P>0,05)$ por los tratamientos (piso y pastoreo), debido quizás a que ya existían jerarquías en los grupos de aves.

Al agrupar las conductas evaluadas, se observa que el bienestar animal fue negativamente afectado (estiramiento de patas y alas e interacción con otras aves), presentando tiempos menores $(\mathrm{P}<0.05)$ para su expresión, o ausencia de las mismas, como ocurrió con la acciones de escarbar y caminar, imposibilitadas por la permanencia en jaulas y la carencia de una cama o el piso de tierra. CASTAÑEDA y GÓMEZ (2011) observaron que las gallinas confinadas en jaula presentaron movimientos anormales repetitivamente. Algo que queda demostrado con esta investigación donde se observó que existen conductas que son imposibles de realizar por estos animales debido al poco espacio con que cuentan.

Las gallinas manejadas en pastoreo dedicaron más tiempo $(P<0.05)$ a estirar las alas, comportamiento que les permite el espacio que disponen para sus actividades diarias, con relación a los demás manejos. BARBOSA (2004) en su investigación determinó que las gallinas criadas en jaulas tiene la dificultad de realizar conductas como estirar alas y agitar plumas gracias a la alta densidad en que se encuentran, más aun desde el punto de vista del bienestar animal afirma que la expresión de estos movimientos sirve como referencia a la hora de evaluar las instalaciones donde se tienen las aves y la falta de estos podría tener las aves en condiciones de estrés.

Las aves manejadas en piso dedicaron más tiempo $(P<0.05)$ a darse "baños" con la cama, comportamiento que se debe a disponibilidad de 
tener una cama de viruta de madera. BARBOSA (2004), quien comparó gallinas de jaula con las de piso, estableció que la cama favorece a las aves a la hora de realizar baños de arena el cual es un comportamiento natural en las aves.

El plumaje de las aves enjauladas se notó deteriorado en comparación a las gallinas de los otros sistemas de manejo. APPLEBY et al. (2004) afirma que las gallinas enjauladas no tienen el suficiente espacio para acicalarse con el pico, darse baños de arena, sacudirse y batir alas, comportamientos necesarios para tener un buen plumaje. Teniendo en cuenta que las aves en jaula durante este trabajo no tuvieron la posibilidad de realizar baños con arena, lo cual no favorece al plumaje de las mismas.

Se concluye que bajo las condiciones del estudio, el manejo de gallinas en piso promovió un mejor desempeño productivo y facilitó la expresión de conductas propias de estas aves, mientras que el manejo en jaulas afectó negativamente el desempeño de las aves, la apariencia y el bienestar de las mismas. El manejo ofrecido en pastoreo propició la realización de conductas propias de las aves pero con un desempeño productivo inferior.

\section{Referencias}

A.O.A.C - Association of Official Agricultural Chemists. Oficial methods of analysis. 15th ed. Washington DC, USA; 1990.

APPLEBY, M.; MENCH, J.; HUGHES, B. 2004. Poultry Behaviour and Welfare Wallingford, England: CABI Publishing, p.46.

BALLINA. A. 2008. Manejo eficiente de gallinas de patio. Instituto Nicaragüense de Tecnología Agropecuaria (INTA). Instituto Nacional Tecnológico (INATEC).

BARBOSA, F. 2004. Avaliação do bem-estar de aves poedeiras em diferentes sistemas de produção e condições ambientais, utilizando análise de imagens. Dissertação (Mestrado) - Escola Superior de Agricultura Luiz de Queiroz. Piracicaba.

BASTMAN, M.; RUIS M.; HEIJMANS, J.; MIDDELKOOP, K. 2011. Poultry signals: A practical guide for bird focused poultry farming. Roodd Bont Publishers. Holanda. 112p.

CASTAÑEDA, C.; GÓMEZ, J. 2011. Evaluación del bienestar animal y comparación de los parámetros productivos en gallinas ponedoras de la lineaHy-line Brown en tres modelos de producción piso, jaula y pastoreo. Facultad de Ciencias Agropecuarias. Universidad de la Salle. Bogotá, Cundinamarca, Colombia.

CASTELLÓN, E.; DURAN, A.; ESCALADA, G.; FARRÉ, A. FERNÁNDEZ, A. 2013. Influencia de los distintos temas de producción sobre el bienestar de las gallinas ponedoras. Normas mínimas relativas a la protección de las gallinas ponedoras. Facultad de Veterinaria-UAB, Bellaterra. P 72-73.

ESTRADA, E.; CASTRO, F.; PRIETO, J. 2015. Bases sociales y políticas del bienestar animal en la Unión Europea. Ambienta 112(9):68-93.

FEDERACIÓN NACIONAL DE AVICULTORES DE COLOMBIA - FENAVI. FENAVI. (2015). Balance avícola 2015 y expectativas 2016. [Acceso 25 de agosto 2015]. Disponible en: URL: http://www.fenavi. org/index.php?option=com_content.

GARCÍA, R.; BERROCAL, J.; MORENO, L.; FERRÓN, G. 2009. Producción ecológica de gallinas ponedoras. Ed. Junta de Andalucía. España. 117p. 
GARCÍA, R., BERROCAL, J., MORENO, L., FERRÓN, G. 2013. Producción ecológica de gallinas ponedoras. Junta de Andalucía Consejería de Agricultura y Pesca. p.20-41.

HINCAPIÉ J; RODAS R. 2001. Manual de explotación de gallinas ponedoras. Zamorano.

HOLDRIDGE, L.R. 1967. Life zone ecology. Tropical Science Center. San José, Costa Rica. 206 p.

KEELING, L. Behaviour of fowl and other domesticated birds. In:The ethology of domestic animals, an introductory text. Ed. JENSEN, P. CABI Publishig.UK. 2003. Cap. 7. 101-117.

LASCARRO, C. (2014). Gallinas ponedoras aviculture - Sena. Servicio Nacional de Aprendizaje - SENA.

LAY JR. D.C.; FULTON, R.M.; HESTER, P.Y.; KARCHER, D.M.; KJAER, J.B.; MENCH, J.A.; MULLENS, B.A.; NEWBERRY, R.C.; NICOL, C.J.; O'SULLIVAN, N.P.; PORTER, R.E. 2011. Hen welfare in different housing systems. Poultry Science 90(2):278-294.

LI, F.; XU, L.M.; HU, J.W.; ZHANG, Y.Y.; LI, Y.J. 2011. Effect of daily feed intake in laying period on laying performance, egg quality and egg composition of genetically fat and lean lines of chickens. British Poultry Science 52(2):163-168. Disponible en URL: https://www.ncbi.nlm.nih.gov/pubmed/21491238

LOHMANN TIERZUCHT GmbH. 2016. Management Recomendations - Management Guide: Alternative Systems. 80p.

SÁNCHEZ, R.; RAMÍREZ, E.S.; ÁVILA, G.; GARCÍA, H.; MARTÍNEZ, M.; RAMOS, V. 2011. Comparación de patas de ponedoras en piso y jaula. Centro de Enseñanza, Investigación y Extensión en Producción Avícola (CEIEPAV), FMVZ-UNAM, XXXVI Convención Anual ANECA, Ixtapa- Zihuatanejo, México.

SOSNÓWKA-CZAJKA, E.; HERBUT, E.; SKOMORUCHA, I. 2010. Effect of different housing systems on productivity and welfare of laying hens. Annals of Animal Science 10(4):349-360. http://www.izoo. krakow.pl/czasopisma/annals/2010/4/art02.pdf

WELFARE QUALITY® Assessment Protocol for laying hens. 2009. Results assessment of 122 flocks. Report 589.Publisher. Wageningen UR Livestock Research P.O. Lelystad.

YILMEZ DIKMEN, B.; IPEK, A.; ŞAHAN, Ü.; PETEK, M.; SÖZCÜ, A. 2016. Egg production and welfare of laying hens kept in different housing systems (conventional, enriched cage, and free range). The Journal of Applied Poultry Research 95(7):1564-1572. Disponible en URL: http://ps.oxfordjournals.org/ content/95/7/1564.abstract 